\title{
EFFECT OF NON-SPHERICITY OF GRAVITATIONAL FIELD OF THE ASTEROID ON THE ITS SATELLITES ORBITS EVOLUTION
}

\author{
V.V. Troianskyi \\ Astronomical Observatory, I.I. Mechnikov Odessa National University \\ Shevchenko Park, 65014, Odessa, Ukraine,v.troianskyi@onu.edu.ua
}

ABSTRACT. Lately it has become possible to sufficiently precise definition of the physical and geometric characteristics of asteroid systems using radar observations (http://echo.jpl.nasa.gov/ lance/binary.neas.html), which allowed calculating the asymmetry of the massive component of double and multiple asteroids for studying the dynamics of motion of their satellites.

Key words: asteroid, gravitational field asteroid, satellites asteroids, evolution orbits.

\section{Gravity field asteroids}

If we interpret the gravitational field of the asteroid in the zero approximation as the gravitational field of a sphere with a symmetrical distribution of the density of its gravitational potential will have a very simple form:

$$
U=\frac{\mu}{r}
$$

where $r$ - distance from the center of the sphere, $\mu-$ gravitational constant.

In the case of the spheroid model, the gravitational potential in a spherical coordinates can be represented as an expansion using Legendre polynomial the 2nd order (Aleksandrov et al., 2004):

$$
U=\frac{\mu}{r}\left[1-\left(\frac{a}{r}\right)^{2} J_{2} P_{2}(\cos \theta)\right],
$$

where $a$ - equatorial radius,

$$
J_{2}=\frac{C-A}{M a^{2}}
$$

where $M-$ the mass of the asteroid. The value of $J_{2}$, characterizes a deviation of the gravitational field of the asteroid from the gravitational field of the sphere.

$$
P_{2}(\cos \theta)=\frac{3}{2} \cos ^{2} \theta-\frac{1}{2}
$$

- the 2 nd order Legendre polynomial, $\theta$ - polar angle of the asteroid's satellite,

$$
C=\int\left(x^{2}+y^{2}\right) d M=\rho\left(\frac{1}{3} x^{3} y z+\frac{1}{3} y^{3} x z\right)
$$

- the moment of inertia relative to the polar axis of the asteroid,

$$
A=\int\left(y^{2}+z^{2}\right) d M=\rho\left(\frac{1}{3} y^{3} x z+\frac{1}{3} z^{3} x y\right)
$$

- moment of inertia relative to the equatorial axis of the asteroid, $x, y, z$ - sizes of the asteroid on the axes of inertia.

$$
\rho(r, \theta, \varphi)=\text { const }
$$

- the density of the asteroid.

Similar model was used to study the dynamics of motion of the triple asteroid system (45) Eugenia (Marchis et al., 2010).

\section{Calculating the magnitude of $J_{2}$}

For calculation of coefficients of the second zonal harmonic, we used the formulas (3) - (6). The following

\begin{tabular}{|c|c|c|c|c|c|c|}
\hline Asteroids systems & $\operatorname{Alpha} J_{2}$ & Error & Beta $J_{2}$ & Error & Gamma $J_{2}$ & Error \\
\hline (1862)Apollo & -0.03489 & $\begin{array}{l}+0.00277 \\
-0.00308\end{array}$ & -0.00362 & $\begin{array}{l}+0.00340 \\
-3.70548\end{array}$ & - & - \\
\hline (66391)1999KW4 & -0.02795 & $\begin{array}{l}+0.00096 \\
-0.00126\end{array}$ & -0.05840 & $\begin{array}{l}+0,01473 \\
-0,02112\end{array}$ & - & - \\
\hline (136617)1994CC & -0.01339 & $\begin{array}{l}+0.00019 \\
-0.00001\end{array}$ & -0.00881 & $\begin{array}{l}+0.00609 \\
-0.03239\end{array}$ & -0.00729 & $\begin{array}{l}+0.00580 \\
-0.00692\end{array}$ \\
\hline (175706)1996FG3 & 0.05412 & $\begin{array}{l}+0.00455 \\
-0.01010\end{array}$ & -0.00993 & $\begin{array}{l}+0.00527 \\
-0.01429\end{array}$ & - & - \\
\hline (276049)2002CE26 & -0.01880 & $\begin{array}{l}+0.00258 \\
-0.00397\end{array}$ & -0.00552 & $\begin{array}{l}+0.00421 \\
-0.03640\end{array}$ & - & - \\
\hline
\end{tabular}
Near-Earth asteroid systems were selected: (1862) Apollo, (66391) 1999KW4, (136617) 1994CC, (175706) 1996FG3, (276049) 2002CE26. For these systems, their physical parameters are known the most precisely (www.johnstonsarchive.net).

Table 1 . The coefficients of the second zonal harmonic $J_{2}$ for selected asteroid systems. 
Table 1 shows the results of calculation of the coefficients $J_{2}$ and their errors for the five selected asteroid systems. In accordance with the generally accepted rules, the components of the asteroid system are identified as follows: Alpha - the main asteroid, and then, in order of the discovering of its satellites Beta, Gamma, etc.

Obtained results are used for modeling of gravitational potential of the main asteroids of asteroid systems. Due to significant error in the determination of $J_{2}$ we consider asteroid satellites as material points. Differential equations of the asteroid's satellite motion in the inertial coordinate system can be expressed as:

$$
\frac{d^{2} \vec{r}}{d t^{2}}-\operatorname{grad} \vec{U}=0
$$

where $\vec{r}=(x, y, z)-$ position vector, $t$ - time, $U-$ the gravitational potential due to attraction of the main asteroid.

For the integration of differential equation of motion we used the method of Everhart 15th order (Bazyey et al., 2009).

\section{Accuracy of integration}

When using the numerical integration of equation of motion, the control of precision of the results of the integration is a must. Precision of integration of asteroid satellites positions of "Beta" (66391)1999KW4, "Beta" (136617)1994CC, "Gamma" (136617)1994CC do not exceed $1.5 \times 10^{-7}$ meters. Magnitude of error is obtained by method of the forward and reverse integration of the motion in the 100 years interval.

\section{Evolution of the orbit of asteroid satellite}

For asteroid systems (66391) 1999KW4 and (136617) 1994CC orbital parameters are known

most precisely (www.johnstonsarchive.net). In Table 2 are given Kepler elements of orbits for satellites in these systems.

As a result of integration of double and multiple asteroid systems, we have obtained changes in orbital elements (solid line) of satellites considering the error (dashed line). In Fig. 1 - Fig. 9 we shows the changes in the elements of the satellites orbits considering disturbances from the compression of the main component of the system. On these figures, we conclude that the ageold changes of semi-major axis, eccentricity and inclination of orbits are hot detected.

Table 2. Kepler elements of orbits of asteroids satellites.

\begin{tabular}{|l|l|l|l|l|l|c|}
\hline \multicolumn{1}{|c|}{ Satellite } & \multicolumn{1}{c|}{ a, meter } & \multicolumn{1}{c|}{ e } & \multicolumn{1}{c|}{ i, degrees } & \multicolumn{1}{c|}{$\omega$, degrees } & $\Omega$, degrees & Epoch, JD \\
\hline "Beta" (66391)1999KW4 & 2540 & 0.0004 & 156.1 & 319.7 & 105.4 & 2456800.5 \\
\hline "Beta" (136617)1994CC & 1720 & 0.002 & 83.3 & 130.9 & 59.2 & 2454994.5 \\
\hline "Gamma" (136617)1994CC & 6100 & 0.19 & 71.7 & 96.2 & 96.2 & 2454994.5 \\
\hline
\end{tabular}

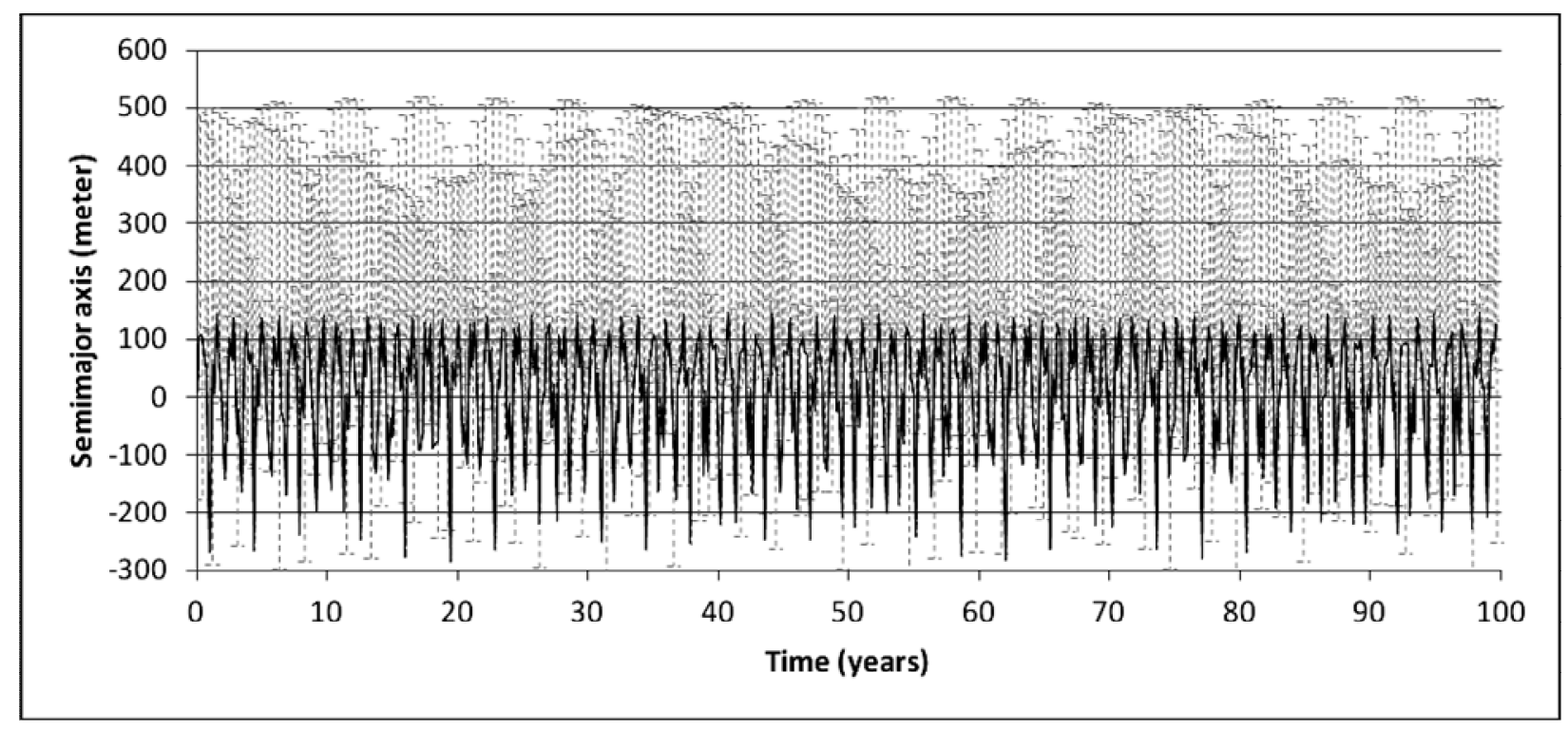

Figure 1: Change of semimajor axis of the orbit of satellite "Beta" of the asteroid system (66391)1999KW4. 


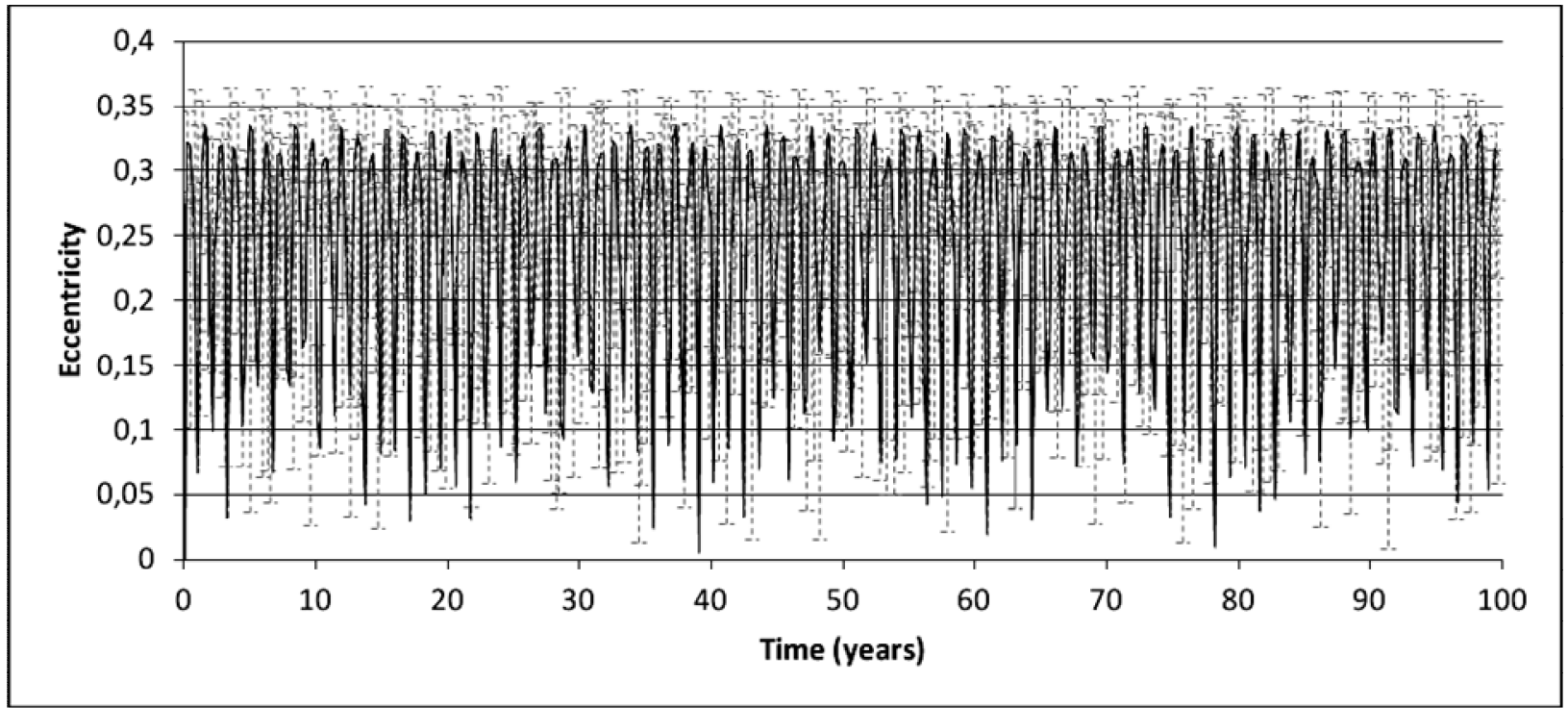

Figure 2: Change of eccentricity of the orbit of satellite "Beta" of the asteroid system (66391)1999KW4.

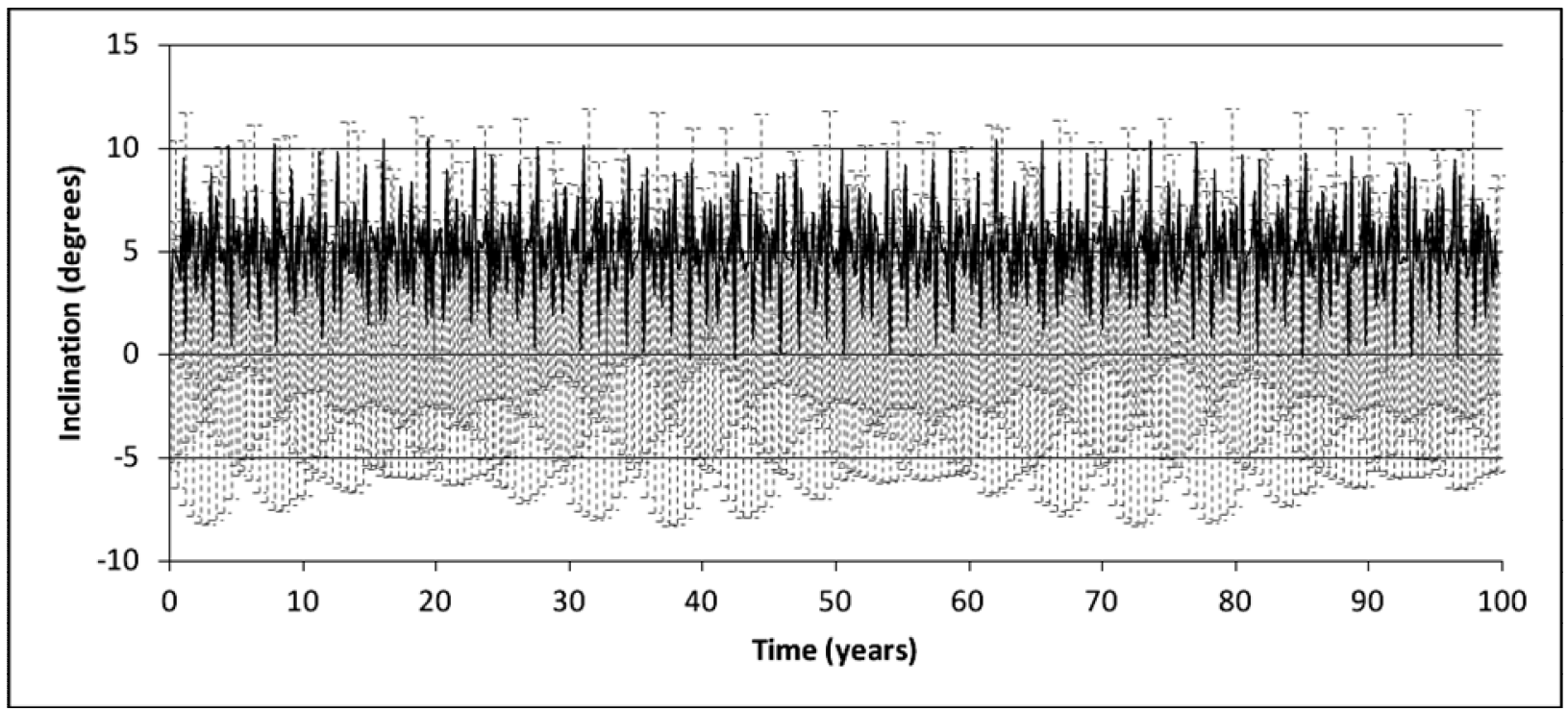

Figure 3: Change of inclination of the orbit of satellite "Beta" of the asteroid system (66391)1999KW4.

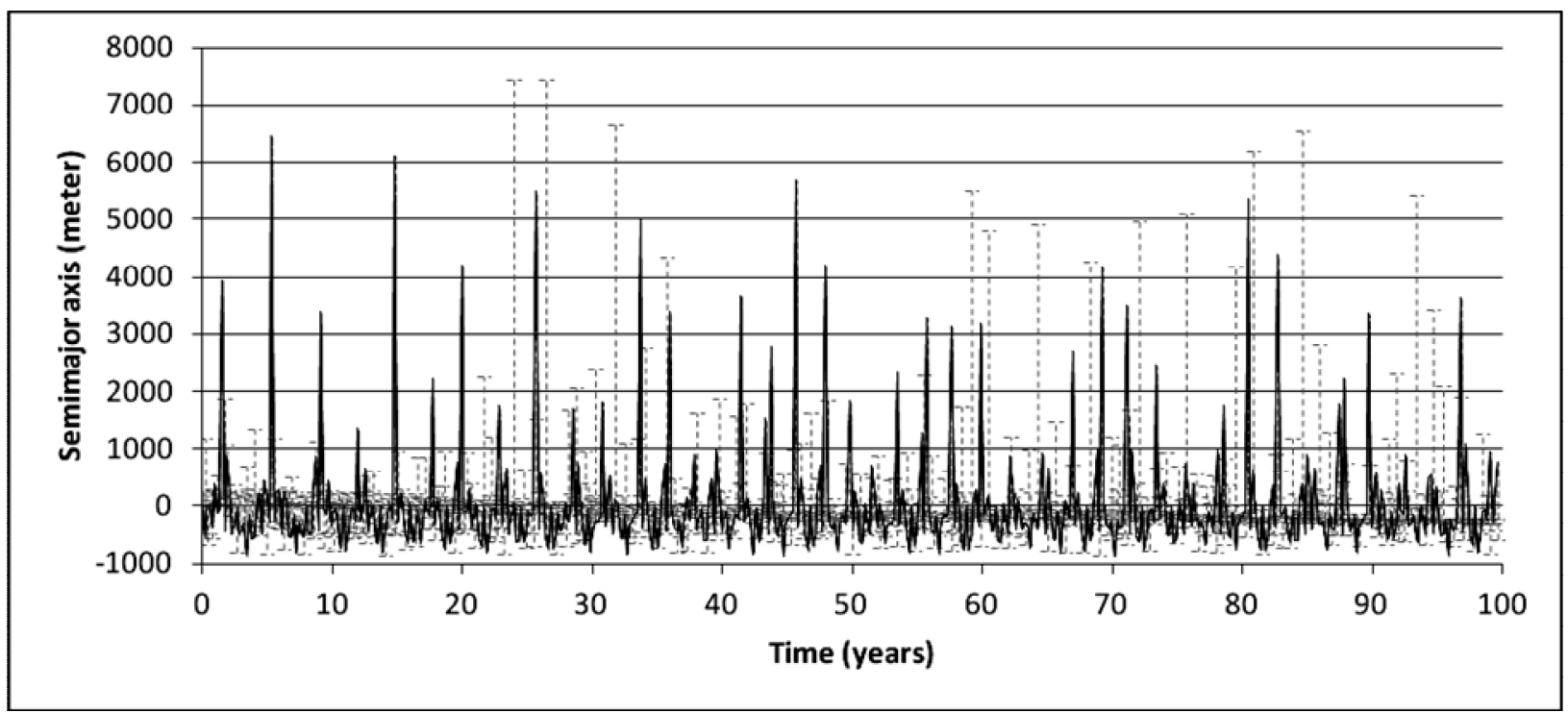

Figure 4: Change of semimajor axis of the orbit of satellite "Beta" of the asteroid system (136617)1994CC. 


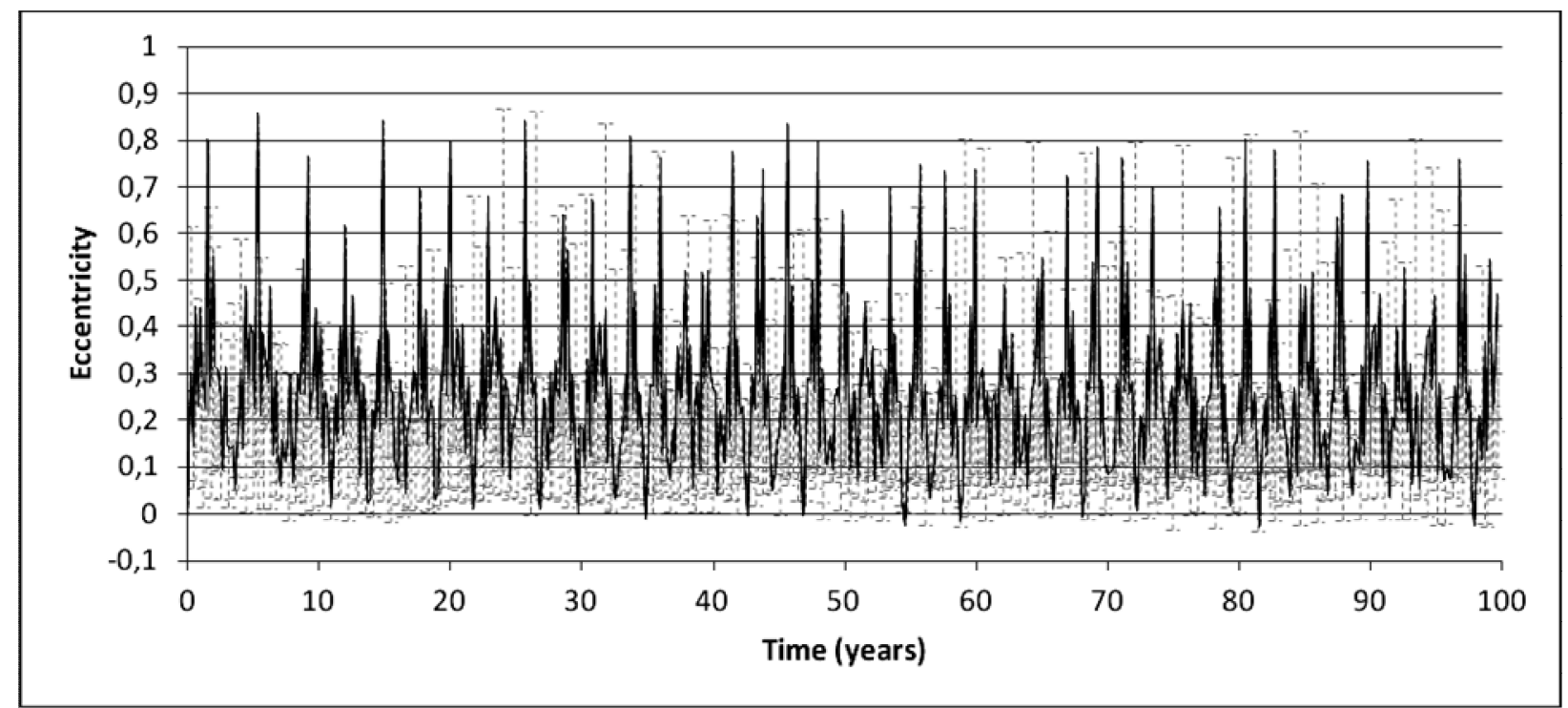

Figure 5: Change of eccentricity of the orbit of satellite "Beta" of the asteroid system (136617)1994CC.

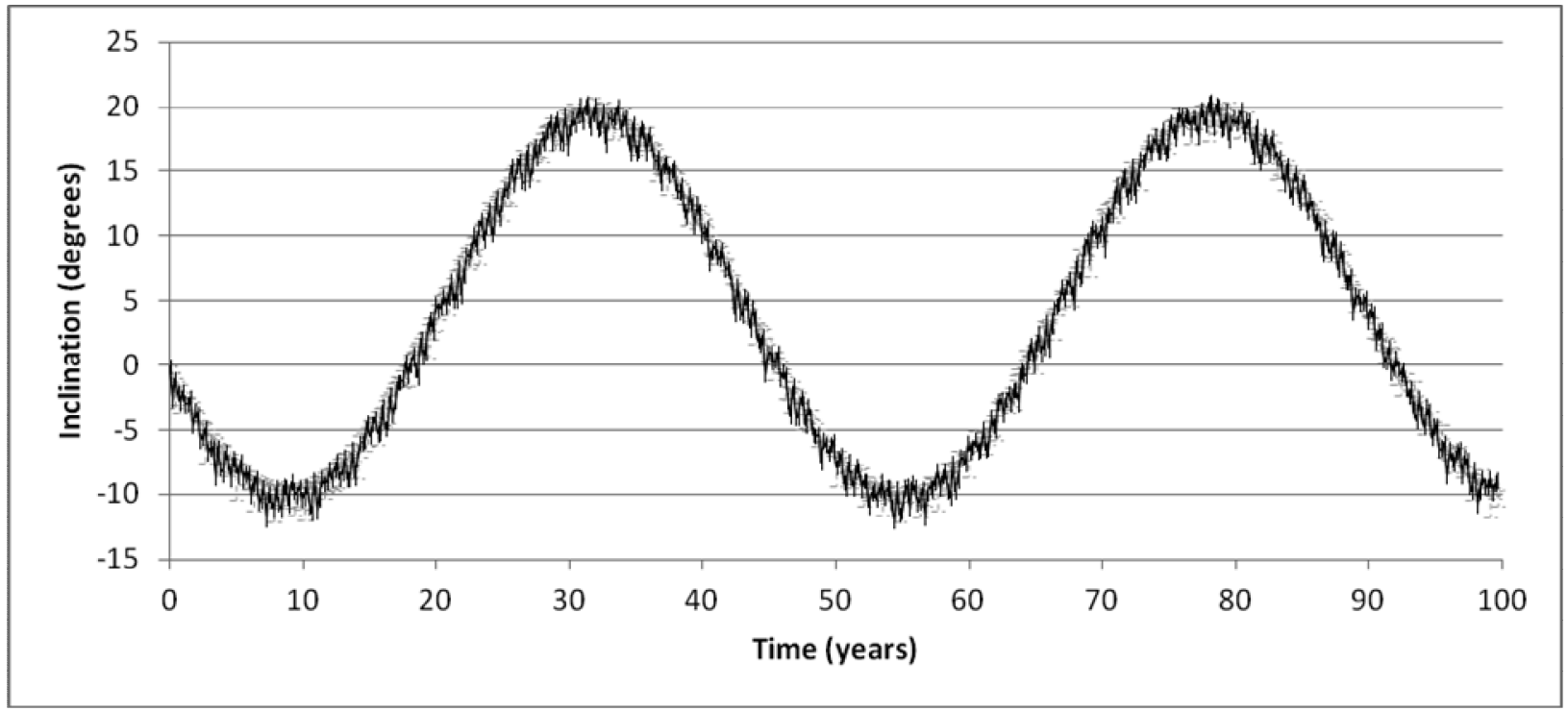

Figure 6: Change of inclination of the orbit of satellite "Beta" of the asteroid system (136617)1994CC.

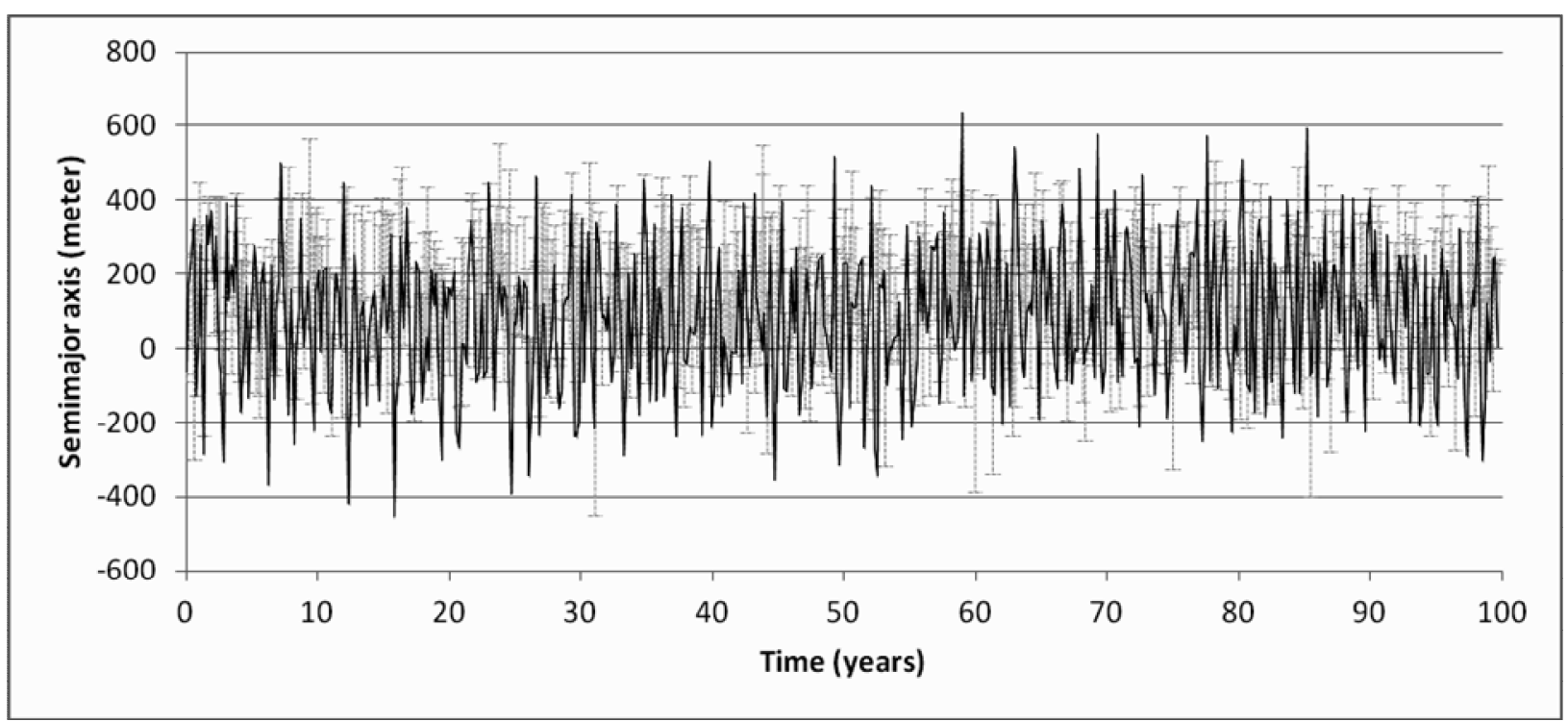

Figure 7: Change of semimajor axis of the orbit of satellite "Gamma" of the asteroid system (136617)1994CC. 


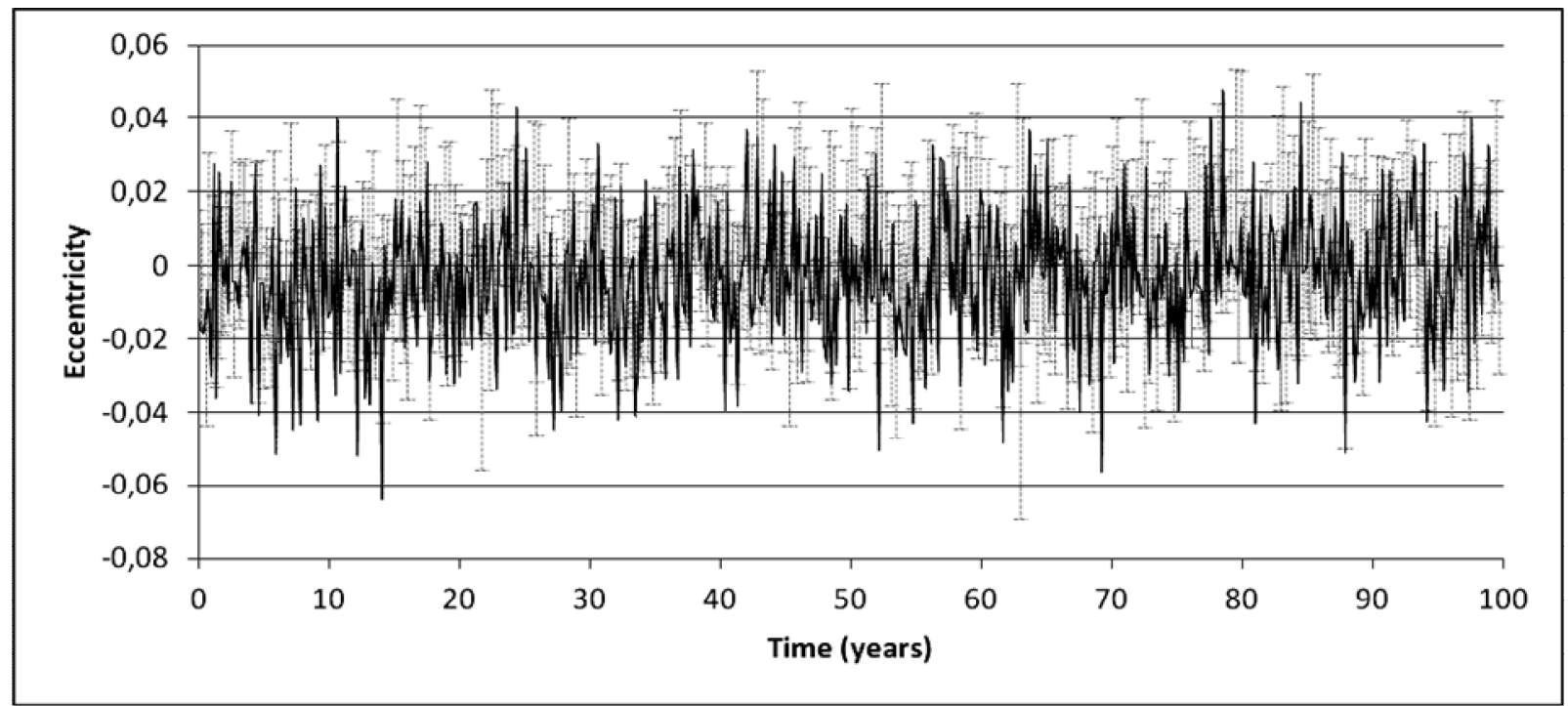

Figure 8: Change of eccentricity of the orbit of satellite "Gamma" of the asteroid system (136617)1994CC.

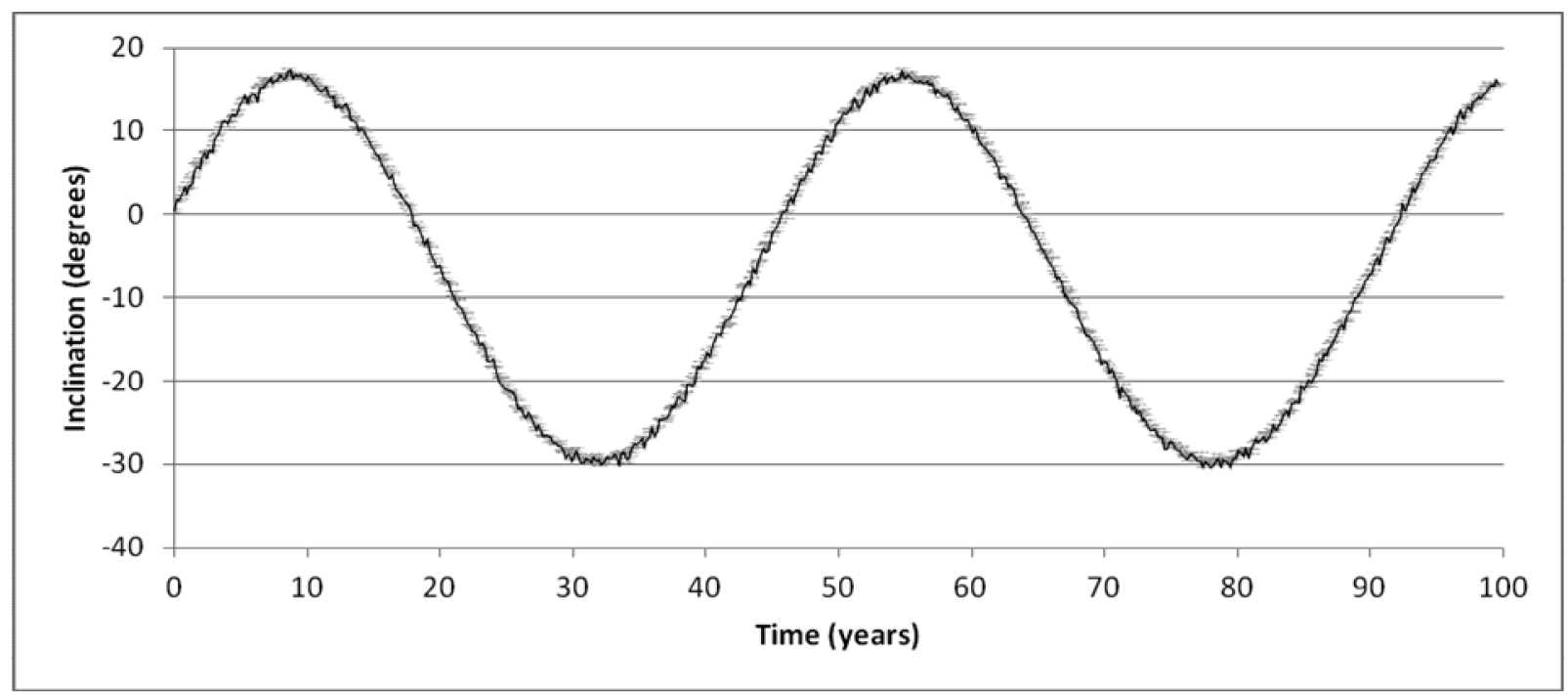

Figure 9: Change of inclination of the orbit of satellite "Gamma" of the asteroid system (136617)1994CC.

\section{Conclusion}

Using the known physical and geometrical parameters of the components of selected double and multiple asteroid systems we have determined the value of the second zonal. As it is seen from Table 1 error in determining of the second zonal harmonic of asteroids satellites is high due of inaccurate initial data.

We also perform the integration of equations of motion of the satellite "Beta" of the asteroid (66391) 1999KW4 in Cartesian coordinates considering the compression of the main asteroid. The modeling showed that the semimajor axis, eccentricity and inclination of the orbit of the satellite change periodically with amplitudes $\approx 216$ meters, $\approx 0.168$ and $\approx 5.38^{\circ}$, respectively.

In the asteroid system (136617) 1994CC satellite "Beta" is located very close to the surface of the central asteroid (about 650 meters), so the impact on him of the asymmetry of the gravitational field is very high. This is illustrated by Fig. 5 - Fig. 6: semimajor axis, eccentricity and inclination of the orbit of the satellite change periodically with amplitudes of $\approx 3235 \mathrm{~m}, \approx 0.429, \approx$ $10.2^{\circ}$, respectively.
In the same asteroid system satellite "Gamma", is much further and therefore the influence on him of the asymmetry of the gravitational field affects not so much, semimajor axis, eccentricity and inclination of the orbit of the satellite change periodically with amplitudes $\approx 318$ meters, $\approx 0.024, \approx 8.7^{\circ}$, respectively. For both satellites the period of changes in orbital inclination of 45.7 years is clearly observed.

Numerical modeling of the motion of the components of double and multiple asteroid systems shows the need to consider the asymmetry of the gravitational field of the main asteroid to describe the dynamics of movement and evolution of satellite orbits of asteroids. This task requires further and more detailed investigation.

\section{References}

Aleksandrov V.Yu.: 2004, Nebesna mehanika (in Ukrainian). Bazyey A.A., Kara I.V.: 2009, Visnyk Astronomichnoyi shkoly, 6/2, 155 (in Ukrainian).

Marchis F., Lainey V., Descamps P. et al.: 2010, Icarus, 210, 635 . 